\title{
BEHAVIOR OF COUNTABLY GENERATED PURE-PROJECTIVE MODULES
}

\author{
Goro Azlmaya
}

Dedicated to the memory of Professor Pere Menal

\begin{abstract}
We first prove that every countably presented module is a pure epimorphic image of a countably generated pure-projective module, and by using this we show that if cvery countably generated pure-projective module is pure-injective then every module is pure-injective, while if in any countably generated pure-projective module cvery countably generated pure-projective pure submodvle is a direct summand then every module is pure-projective.
\end{abstract}

Let $R$ be a ring. A left $R$-module $M$ is called pure-projective if every pure epimorphism onto $M$ splits. As is well-known, $M$ is pure-projective if and only if $M$ is a direct summand of a direct sum of finitely presented left $R$-modules. On the other hand, $M$ is called pure-injective if for any left $R$-module in which $M$ is embedded as a pure submodule $M$ is always a direct summand of it. We call $R$ a left pure-semisimple ring or a ring of left pure global dimension zero if it satisfies the following obviously equivalent conditions: (1) every left $R$-module is pure-projective, (2) every left $R$-module is pure-injective, (3) for any left $R$-module $M$ every pure submodule of $M$ is a direct summand of $M$. It has been known that every left pure-semisimple ring is a left Artinian ring and besides lcft pure-semisimple rings are characterized as those rings $R$ for which the following equivalent conditions hold: (4) every left $R$-module is a direct sum of finitcly generated submodules, (5) every left $R$-module is a direct sum of indecomposable submodules, (6) every indecomposable left $R$-module is finitely presented $([\mathbf{2}],[\mathbf{3}],[\mathbf{8}],[\mathbf{1 0}])$. The author has then shown in [1] that even if we replace the terms every and any in (1), (2), (3) and (6) by the terms every countably generated and any countably generated respectively we still have conditions each equivalent to the left 
pure-semisimplicity of $R$. Now in this paper we shall give further characterizations of pure-semisimple rings in terms of countably generated pure-projective modules. In order to confirm these characterizations we need to combine Simson's theorem in [6] that if every countably presented left $R$-module is pure-projective then $R$ is left pure-semisimple with the following crucial proposition, which refines the known Warfield's theorem in [7] that every module is a pure epimorphic image of a direct sum of finitely presented modules and where a module is called countably presented if it is isomorphic to the factor module of a countably generated free module modulo a countably gencrated submodule:

Proposition 1. Let $M$ be a countably presented left $R$-module. Then there exists a left $R$-module $P$ which is a countable direct sum of finitely presented left $R$-modules and has a pure epimorphism $P \rightarrow M$.

Proof: Since $M$ is countably presented, there exist a (infinite-) countably generated free left $R$-module $F$, a countably generated submodule $G$ of $F$ and an epimorphism $\varphi: F \rightarrow M$ whose kernel is $G$. Let $u_{1}, u_{2}, u_{3}, \ldots$ be a countable free basis of $F$ and $v_{1}, v_{2}, v_{3}, \ldots$ a countable generators of $G$. For each positive integer $n$, we denote by $F_{n}$ the finitely generated free submodule $R u_{1} \oplus R u_{2} \oplus \cdots \oplus R u_{n}$ of $F$ and by $G_{n}$ the finitely generated submodule $R v_{1}+R v_{2}+\cdots+R v_{n}$ of $G$. Then clearly we have $F=\sum_{n=1}^{\infty} F_{n}$ and $G=\sum_{n=1}^{\infty} G_{n}$. Morcover, for each $n$, there is an $m$ such that $G_{n} \subset F_{n n}$. Let $l(n)$ be the least one among such $m$ 's, and let $m(n)=\max (l(n), n)$. Then, it is obvious that $G_{n} \subset F_{m(n)}$ for every $n$ and $F=\sum_{n=1}^{\infty} F_{m(n)}$.

Consider now countable (outer) direct sums $S=\bigoplus_{n=1}^{\infty} F_{m(n)}$ and $T=$ $\bigoplus_{n=1}^{\infty} G_{n}$. Then $T$ is a submodule of $S$. For each $n$, wo denote by $q_{n}$ the $n$-th canonical embedding $F_{m(n)} \rightarrow S$, i.e., $q_{n}(x)$, for $x \in F_{m(n)}$, is the element of $S$ whose $n$-th entry is $x$ and other entries are all 0 . Clearly the restriction of $q_{n}$ to the submodule $G_{n}$ of $F_{m(n)}$ is the $n$-th canonical embedding $G_{n} \rightarrow T$. Now the factor modulc $F_{n n(n)} / G_{n}$ is finitely presented for each $n$. Let $P=\bigoplus_{n=1}\left(F_{m(n)} / G_{n}\right)$ be the countable direct sum. Then the natural epimorphisms $F_{m(n)} \rightarrow F_{m(n)} / G_{n}$ together define an epimorphism $\psi: S \rightarrow P$ whose kernel is $T$. If we next associate 
each $s \in S$ with the sum $\sum_{n=1}^{\infty} s_{n}$ in $F$, where $s_{n}\left(\epsilon F_{m(n)}\right)$ is the $n$-th entry of $s$, then we have an epimorphism $f: S \rightarrow F$. The restriction of $f$ to $G$ gives clearly an epimorphism $g: T \rightarrow G$, and so we have that $\varphi(f(T))=\varphi(g(T))=\varphi(G)=0$. Since $T$ is the kernel of $\psi$, this implies that an epimorphism $h: P \rightarrow M$ is well-defined so that $h \circ \psi=\varphi \circ f$.

We now show that $h$ is a pure epimorphism. Let $E$ be a finitely presented left $R$-module. Thus there exist a finitely generated free left $R$-module $L$ and an cpimorphism $\pi: L \rightarrow E$ whose kernel $K$ is finitely generated. Let $\alpha: E \rightarrow M$ be a homomorphism. Consider the product $\alpha \circ \pi: L \rightarrow M$. Since $L$ is projective, there must exist a homomorphism $\beta: L \rightarrow F$ such that $\varphi \circ \beta=\alpha \circ \pi$. From this follows that $\varphi(\beta(K))=$ $\alpha(\pi(K))=0$ and so $\beta(K) \subset G$, the kernel of $\varphi$. Since both $L$ and $K$ are finitely generated, their homomorphic images $\beta(L)$ and $\beta(K)$ are finitely generated submodules of $F$ and $G$ respectively. Therefore we have $\beta(L) \subset F_{m(n)}$ and $\beta(K) \subset G_{n}$ for sufficiently large $n$. We fix such an $n$, and define the homomorphism $\gamma: L \rightarrow S$ by $\gamma=q_{n} \circ \beta$. Then we have $f \circ \gamma=f \circ q_{n} \circ \beta$, but since $f \circ q_{n}$ is clearly the inclusion map $F_{m(n z)} \rightarrow F$ it follows that $f \circ \gamma=\beta$. On the other hand, that $\beta(K) \subset G_{n}$ implies that $\gamma(K)=q_{n}(\beta(K)) \subset q_{n}\left(G_{n}\right) \subset T$ and therefore $\psi(\gamma(K)) \subset \psi(T)=0$, i.e., $K$ is contained in the kernel of $\psi \circ \gamma: L \rightarrow P$. This shows that a homomorphism $\delta: E \rightarrow P$ is well-defined so that $\delta \circ \pi=\psi \circ \gamma$. We have then $h \circ \delta \circ \pi=h \circ \psi \circ \gamma=\varphi \circ f \circ \gamma=\varphi \circ \beta=\alpha \circ \pi$, but since $\pi: L \rightarrow E$ is an epimorphism we know that $h \circ \delta=\alpha$. This is true for cvery finitely presented module $E$ and for every homomorphism $\alpha: E \rightarrow M$, and thus it is proved that $h$ is a pure epimorphism.

Theorem 2. $R$ is left pure-semisimple if and only if every countably generated pure-projective left $R$-module is pure-injective.

Proof: Clearly we need only prove the if part. Let $M$ be any countably presented left $R$-modulc. Then by the preceding proposition there exist a countable direct sum $P$ of finitely presented left $R$-modules and an epimorphism $h: P \rightarrow M$ whose kernel $Q$ is a pure submodule of $P$. Now the direct. sum $P^{(N)}, N$ being the set of all natural numbers, of the countable number of copies of $P$ is also a countable direct sum of finitely presented left $R$-modules and hence is countably generated and pure-projective. Thus by our assumption $P^{(N)}$ is pure-injective, or equivalently, $P$ is $\sum$-pure-injective. Therefore the purc submodule $Q$ of $P$ is a direct summand of $P([9$, p. 1100$],[1$, Prop. 3.5]), which means that $M$ can be embedded into $P$ as a direct summand and therefore $M$ is pure-projective too. Thus it lums out that every countably presented 
left $R$-module is pure-projective. It follows from Simson [6, Th. 6.3] that $R$ is left pure-semisimple.

As is easily scen, cvery countable direct sum of countably presented modules is countably presented too and so in particular every countable direct sum of finitely presented modules is countably presented. Therefore, from the preceding theorem we can derive the following, which is however regarded as a dual of the above referred Simson's theorem:

Corollary 3. $R$ is left pure-semisimple if and only if every countably presented left $R$-module is pure-injective.

Now the following is to refine the equivalence of the conditions (2) and (6) in $[1$, Th. 4.5]:

Theorem 3. $R$ is left pure-semisimple if and only if for any countably generated pure-projective left $R$-module $P$ every countobly generated pure-projective pure submodule of $P$ is a direct summand of $P$.

Proof: We need only prove the if part too. Let $M$ be a countably presented left $R$-module. This means that there exist a countably generated free left $R$-module $F$ and an epimorphism $\varphi: F \rightarrow M$ whose kernel $G$ is countably generated. On the other hand, by Proposition 1 , there exist a countable direct sum $P$ of finitely presented left $R$-modules and an epimorphism $h: P \rightarrow M$ whose kernel $Q$ is pure in $P$. We shall show that $Q$ is countably generated too. For, since $F$ is projective there is a homomorphism $f: F \rightarrow P$ such that $h \circ f=\varphi$. Let $p$ be an element of $P$. Then $h(p)$ is in $M$ and so we can find an $x \in F$ such that $\varphi(x)=h(p)$ whence $h(f(x))=h(p)$, i.e., $h(p-f(x))=0$. Thus we know that $p-f(x)$ is in $Q$ and therefore that $P=f(F)+Q$. On the other hand, if $x$ is in $F$ then the equality $h(f(x))=\varphi(x)$ implies that $f(x)$ is in $Q$ if and only if $x$ is in $G$, and therefore we have $f(F) \cap Q=f(G)$. Thus we know that $Q / f(G) \cong P / f(F)$. But since $P$ is countably generated its homomorphic image $P / f(F)$ whence $Q / f(G)$ is countably generated too, while since $G$ is countably generated its homomorphic image $f(G)$ is also countably generated. From this we can conclude that $Q$ is countably generated.

Now, since $P$ is pure-projective, the countably generated pure submodule $Q$ of $P$ is pure-projective according to Kiclpinski-Simson [4, Cor. 1.5]. (This can also be proved by using the notion of Mittag-Leffler modules as follows: As is well-known, every pure submodule of a pure-projective module is a Mittag-Leffer module, and so $Q$ is a Mittag-Leffler module, i.e., the canonical homomorphism $\left(\prod A_{i}\right) \bigotimes_{R} Q \rightarrow \prod_{R}\left(A_{i} \underset{\bigotimes}{\bigotimes}\right)$ is 
a monomorphism for every family $\left\{A_{i}\right\}$ of right $R$-modules. Since $Q$ is countably generated, $Q$ must be pure-projective by Raynaud-Gruson [5, Part II, Cor. 2.2.2].) By the assumption of our theorem, we know that $Q$ is a direct summand of $P$ and so $M$ can be embedded into $P$ as a direct summand, which implies that $M$ is pure-projective. Thus we have shown that every countably presented left $R$-module is pureprojective, and therefore again by Simson's theorem ([6, Th. 6.3]) $R$ is left pure-semisimple.

In this connection, it is to be pointed out that Theorem 2 is a direct consequence of Theorem 3 . For, let $P$ be a left $R$-module and $Q$ a countably generated pure-projective pure submodule of $P$. Suppose that every countably generated pure-projective left $R$-module is pureinjective. Then $Q$ is a direct summand of $P$. Thus, by Theorem $3, R$ is left pure-semisimple.

Remark. A. Abe has independently obtained Proposition 1 and Theorem 2 too. Indeed, he proves Proposition 1 by using [6, Cor. 2.5] that if a module $M$ is a direct limit of modules $M_{i}$ 's then the canonical epimorphism $\bigoplus_{i} M_{\imath} \rightarrow M$ is pure.

\section{References}

1. G. AzUMAYA, "Countable generatedness version of rings of pure global dimension zero," Representations of algebra, Cambridge University Press, 1992.

2. S. U. CHASE, Direct product of modules, Trans. Amer. Math. Soc. 97 (1960), 457-473.

3. L. Gruson AND C. U. Jensen, Deux applications de la notion de L-dimension, C.R. Acad. Sci. Paris, Sér. A 282 (1976), 23-24.

4. R. KIELPIŃSKI AND D. Simson, On pure homological dimension, Bull. Acad. Polo. Sci., Sér. Sci. Math. Astr. Phys. 23 (1975), 16.

5. M. Raynaud ano L. Gruson, Critères de platitude et de projective, Invent. Math. 13 (1971), 1-89.

6. D. Simson, On pure global dimension of locally finitely presented Grothendieck categories, Fundamenta Math. 96 (1977), 91-116.

7. R. B. WARField, JR., Purity and algebraic compactness for modules, Pacific J. Math. 28 (1969), 699-719.

8. W. ZimmermanN, Einige Charakterisierungen der Ringe, uber denen reine Untermoduln direkt Summanden sind, Sitzungsber, Bayer. Akad. 3 (1972), 77-79. 
9. W. Zimmermann, Rein injektive direkte Summen von Moduln, Comm. Algebra 5 (1977), 1083-1117.

10. B. Zimmermann-Huisgen, Rings whose right modules are direct sums of indecomposable modules, Proc. Amer. Math. Soc. $\mathbf{7 7}$ (1979), 191-197.

Department of Mathematics

Indiana University

Bloomington, IN 47405

U.S.A.

Rebut el 7 de Gener de 1992 